\title{
Chapter 11 \\ Data Analytics of Energy \\ and Compressed Air Flows for Process \\ and Quality Monitoring \\ in Electro-Pneumatic Handling Systems
}

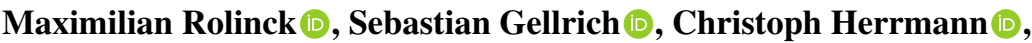 and Sebastian Thiede}

\begin{abstract}
Ensuring constant high process and product quality is one of the decisive competitive factors in today's industrial production. Industry 4.0 by means of the analysis of production data is seen as a viable approach to achieve zero defects production. Due to their sensitive behavior, energy data, which is often acquired independently of the machine control, can provide an adequate data source for quality-related analysis tasks. An analysis based on energy data reduces the effort for accessing machine controls and enables scalability. This paper shows the concept and implementation of a data-driven process and quality monitoring tool based on energy data for an electro-pneumatic handling system.
\end{abstract}

Keywords Cyber Physical Production System - Data Mining - Process and quality monitoring

\subsection{Introduction}

An effective quality control is one of the key challenges in modern production. In order to foster a data-driven quality control, monitoring systems serve as a viable approach. In highly automated production industries electro-pneumatic automation systems gain importance (Statista 2019) and a higher spread in several industries that are sensitive due to hygienic requirements as well as explosive materials (Watter 2017). For this reason, this paper shows an implementation of a data-driven quality control tool for electro-pneumatic automation systems in terms of a Cyber-Physical Production System (CPPS). The CPPS takes the energy data of the electro-pneumatic automation system as input for process and quality monitoring, due to scalability and independence from the machine control. Therefore, background information on

M. Rolinck $(\varangle) \cdot$ S. Gellrich $\cdot$ C. Herrmann $\cdot$ S. Thiede

Chair of Sustainable Manufacturing and Life Cycle Engineering, Institute of Machine Tools and Production Technology (IWF), Technische Universität Braunschweig, Langer Kamp 19b, 38106

Braunschweig, Germany

e-mail: m.rolinck@tu-braunschweig.de

K. S. Sangwan and C. Herrmann (eds.), Enhancing Future Skills and Entrepreneurship,

Sustainable Production, Life Cycle Engineering and Management,

https://doi.org/10.1007/978-3-030-44248-4_11 
CPPS is briefly introduced in Sect. 11.2. On this basis, the concept of a process and quality monitoring tool for an electro-pneumatic handling system is developed in Sect. 11.3. The concept is exemplified within the experimental set-up of the Joint Indo-German Experience Lab at BITS Pilani, India.

\subsection{Research Background}

Facilitated by technological improvements, Industry 4.0 and digital manufacturing are major trends in industry and research ( $\mathrm{Lu} 2017$; Vaidya et al. 2018). CPPS are proposed to be a promising approach to pursue these trends as well as future demands in production (Thiede et al. 2014). According to Thiede et al. CPPS are based on the "implementation of elements from cyber physical systems" which contain "computational and physical capabilities combined with the possibility of human machine interaction" (Thiede et al. 2014). For the assessment and implementation of a CPPS Thiede et al. give an appropriate framework. The framework describes a loop of different key aspects and ranks them in three levels each, as shown in Fig. 11.1. In general, the framework describes a continuous data and information processing between the physical world and the so-called cyber world, which are connected by the elements data acquisition and feedback/control.

To perform analysis in an automated model, data mining gives various possibilities to process data. It can operate six different tasks according to Larose: Description, estimation, prediction, classification, clustering and association. These tasks depend on the intended analysis target and require different data pre-preparation steps (Larose and Larose 2014).

In order to fulfil the introduced six tasks, several methods are available. Different data mining methods perform one or more of the previously named tasks (Chakrabarti

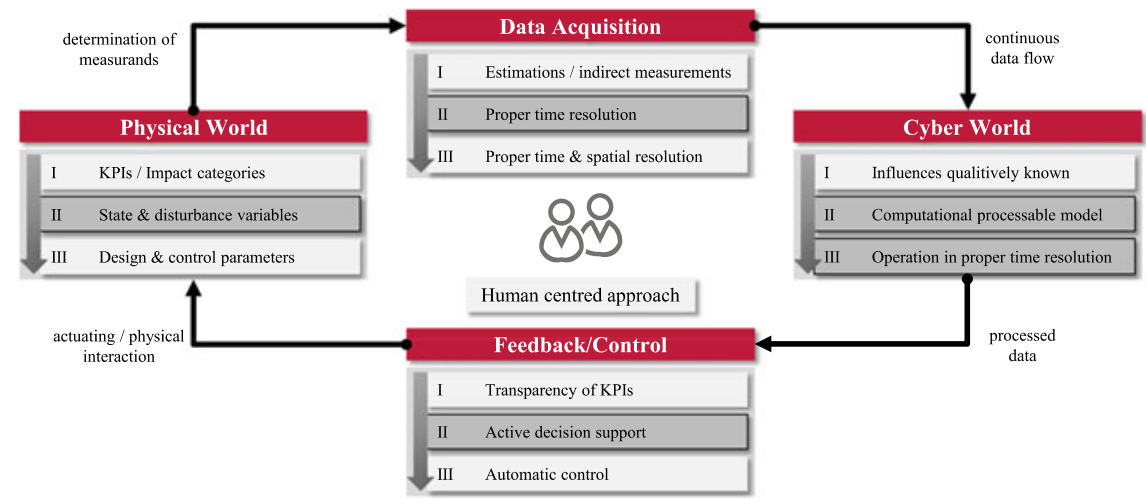

Fig. 11.1 CPPS according to Thiede et al. (2014) with levels highlighted in grey for the concept to be assigned (see Sect. 11.3). Data flows between elements are depicted by black arrows 
et al. 2009). Besides that, the methods can be differentiated into supervised and unsupervised learning. The so called supervised learning is used for estimation, prediction and classification and includes the allocation of target variables to predictor variables. This allocation is also known as labelling. Unsupervised learning is used for clustering, association as well as description and exposes patterns out of unlabeled datasets (Chakrabarti et al. 2009). In order to deploy, for example, a classification task, a supervised learning method that performs a categorical statement based on a labelled dataset is chosen.

\subsection{CPPS-Based Process and Quality Monitoring Tool}

\subsubsection{Concept}

For the conceptual design of a process and quality monitoring tool, the framework of the introduced CPPS is used. In order to provide advanced support to an operator of a machine or production system, accordance with a high level (see Fig. 11.1) in each element is vital. Thus, state variables (II) from the physical world are chosen and gathered at least in proper time resolution (II) in a form of data acquisition. In order to ensure the independence of further data processing from predetermined software solutions or adjustments of machine controls for data access, data gateways can be deployed. Implementing a computational processable model (II) in the cyber world and processing data in proper time resolution (III) results in a higher informational degree about the current state of the machine or production system. The feedback of this information in form of active decision support (II), e.g. alerts or instructions, enables the operator to effectively interact with the physical world.

\subsubsection{Exemplary Implementation}

The concept of a process and quality monitoring tool is exemplary implemented for the learning platform of the Joint Indo-German Experience Lab (JInGEL), funded by the German Academic Exchange Service (DAAD). A Modular Production System (MPS) within the small-scaled production line simulating an assembling process is chosen for this purpose, called pick \& place (p\&p) process in the following. The central task of this station is to pick caps from a chute and to place them on the provided workpieces (see Fig. 11.2, left). Workpieces are distributed to the station one after another on a conveyor belt and transported to the middle of the station. Each piece is hold back by an electro-magnetic driven deflector and meanwhile detected by a sensor. When a workpiece is fixed, the p\&p process starts. A suction cup, mounted on an electro-pneumatic driven handling arm, picks one cap from the chute. After being elevated, it is placed on the workpiece. The assembled product is 

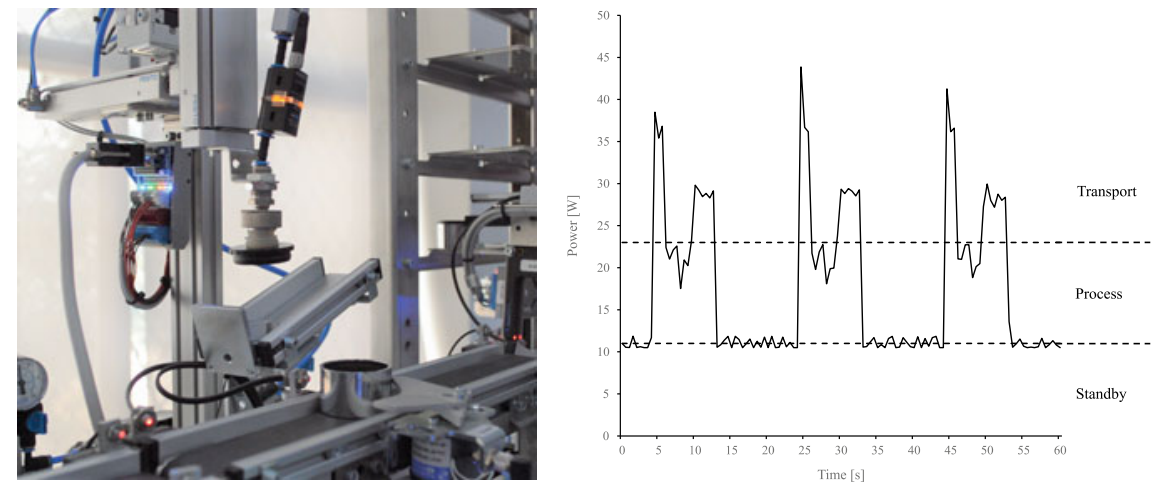

Fig. 11.2 Left p\&p process within the experience lab at BITS Pilani - a cap is elevated from the chute; right classes of power demand of the three p\&p process states

released and transported to the next station. In case that the chute runs empty and no cap is available for the assembling process, the handling arm stops the suction process after detection, but the unassembled workpiece is still transported to the next station. This results in a considerable lack of product quality that should be detected by the monitoring tool. The product is sorted out in one of the following production steps, but the p\&p process runs incorrectly until the operator notices the error and intervenes. In any case, the process runs at a fixed time interval of $20 \mathrm{~s}$. This time stamp serves as a basis for further modeling.

Physical World and Data Acquisition. During the process, the energy state variables current and electrical power as well as compressed air flow are measured. Using a coupler from Beckhoff, the data is converted into E-bus signal representations and transported by an EtherCAT® network. Via the Visual Studio ${ }^{\circledR}$ embedded TwinCAT® software an OPC UA server is installed, which represents a data gateway, enabling data to be accessible for further processing. In order to process data, the flow-based development tool Node-RED is chosen. It provides a platform for functions programming and integration of versatile input and output options. The provided data is stored in a MySQL database which facilitates the computation of the process's energy demand from the measured electrical power. Similar data processing is applied for the compressed air flow giving the average consumption of compressed air. These five state variables enable further analyses that are depicted in the following section. Besides these variables, control parameters are given due to the possibility for an operator to intervene the process by replacing caps or even stopping the process.

Cyber World. For data exploration, data is extracted in a time resolution of $0.5 \mathrm{~s}$. Examining the results for the power demand over three process cycles (Fig. 11.2, right), three different states can be identified. The peaks in the curve classify the demand when a workpiece is transported by the conveyor belt. The transport state is defined for the maximum electrical power demand at the time the conveyor belt is running. The process state is identified by the distinct $p \& p$ process itself when 
the conveyor belt is not running, the workpiece is fixed by the deflector and the electro-pneumatic driven handling arm is manipulating the product. The third state represents the standby mode when a base load at approximately $11 \mathrm{~W}$ is caused by the machine control and lighting.

The process is divided into two different conditions of cap availability, i.e. the quality criteria of the $\mathrm{p} \& \mathrm{p}$ process. Condition 1 is used for a regular process in which caps are available. A process in which caps are missing and the workpiece is therefore not properly assembled is further referred to as condition 2 . The data exploration shows, that no significant difference can be found between both cases in the level of power demand. However, a difference in the time sequence and duration of the process can be observed. In condition 2, the process runs two and a half seconds faster as the handling arms movement is aborted. Regarding the compressed air flow, condition 2 differs from condition 1 with a lower flow rate of about $5 \mathrm{l} / \mathrm{min}$. Consequently, it is determined that condition 1 and condition 2 can be clearly distinguished in their energy consumption and average compressed air flow. The values for energy intensity, which is the energy demand for one process cycle of $20 \mathrm{~s}$, and average compressed air flow over the same interval are depicted in the scatter plot in Fig. 11.3. Clear limits can be found at $350.4 \mathrm{Ws}$ for the electrical energy intensity and $7.95 \mathrm{l} / \mathrm{min}$ for the average compressed air flow, with condition 1 always showing a higher value than condition 2 .

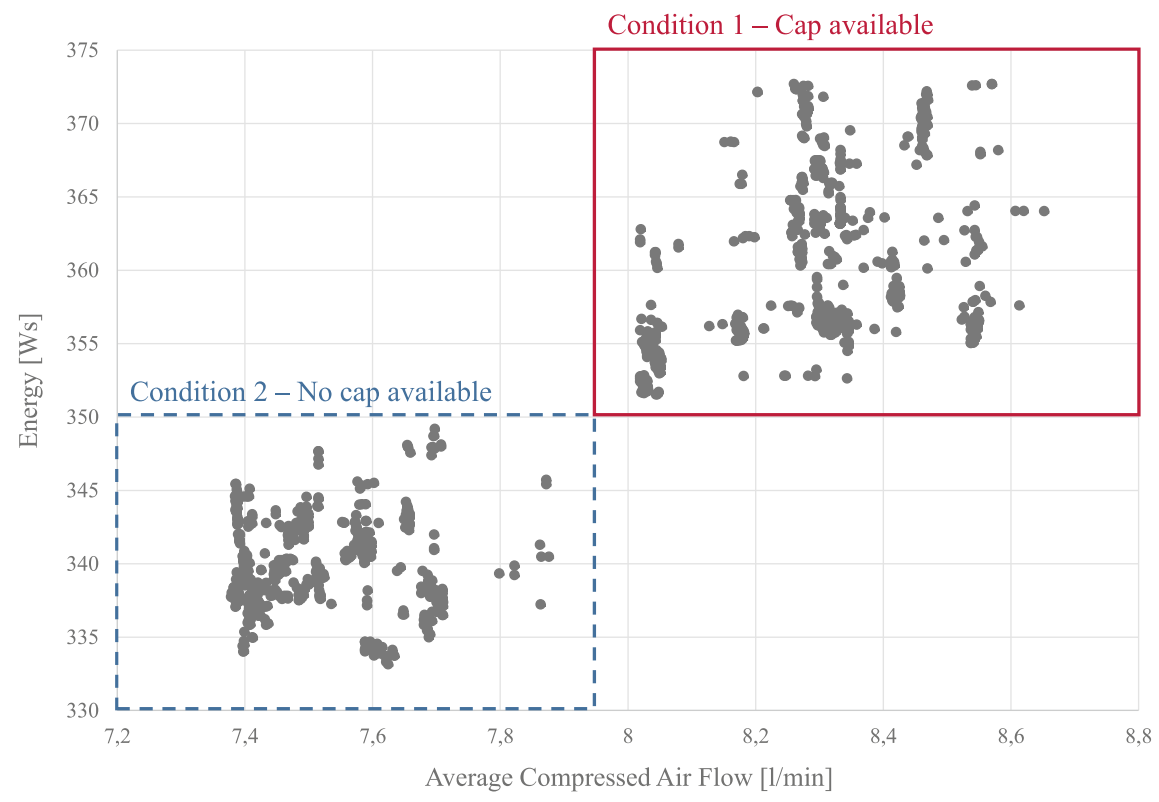

Fig. 11.3 Scatter plot on energy intensity and average compressed air flow for $p \& p$ process. Areas for condition 1 and 2 indicating quality criteria are marked 
Table 11.1 Labelling data arrays for data preparation

\begin{tabular}{l|l|l|l|l|l|l}
\hline Power $(\mathrm{W})$ & $\begin{array}{l}\text { Energy } \\
(\mathrm{Ws})\end{array}$ & Current (A) & $\begin{array}{l}\text { Compr. air } \\
\text { flow } \\
(1 / \mathrm{min})\end{array}$ & $\begin{array}{l}\text { Average air } \\
\text { flow } \\
(1 / \mathrm{min})\end{array}$ & State & Condition \\
\hline 10.93 & 370.68 & 0.09 & 6.52 & 8.46 & Standby & Cap \\
\hline 38.43 & 371.38 & 0.22 & 6.50 & 8.46 & Transport & Cap \\
\hline 21.56 & 368.19 & 0.15 & 22.03 & 8.55 & Process & Cap \\
\hline 33.71 & 337.08 & 0.15 & 17.23 & 7.39 & Process & No cap \\
\hline 28.43 & 337.98 & 0.18 & 6.46 & 7.39 & Transport & No cap \\
\hline 10.65 & 338.03 & 0.09 & 6.58 & 7.39 & Standby & No Cap \\
\hline
\end{tabular}

These findings allow the classification of state variables regarding the state as well as the corresponding quality condition as shown in Table 11.1. For new real-time data, the classification is to be performed through a data mining approach based on supervised learning. In order to train a data mining method, a training data set with over 3,000 arrays of state variables is labelled according to the example shown in Table 11.1. Three supervised learning methods, i.e. random forest, support vector machines and decision trees, are examined. With an overall accuracy of $97.6 \%$ and good results in the F1-Score for the critical distinction of quality condition a decision tree based method is chosen for this use case. The trained model delivers information about the state (transport, process or standby) and the condition (with or without cap) of the process. In a next step, this information shall be provided to the operator via a monitoring tool.

Monitoring and Decision Support. In order to provide the operator with information, a dashboard is designed in Node-RED (Fig. 11.4). Information of two kinds are displayed, on the one hand real-time data about power demand and compressed air flow and on the other hand classifications on the current state and condition of the p\&p system. The classifications allow the assignment of shares of energy intensity due to compressed air flow and electrical energy consumption per cycle and state. This information can be used for a dynamic Energy Value Stream Mapping, as shown in Fig. 11.4. In addition, direct feedback is given to the operator in case of a caps' absence. It appears in the form of a pop-up window which disappears only after an "Ok" button is confirmed and also instructs to replace caps at the p\&p station. The implementation thus provides the operator with a process and quality monitoring tool. In a next step, key performance indicators like an Overall Equipment Effectiveness (OEE) could be implemented due to necessary parameters already being calculated by the system. In the current visualisation the amount of correctly assembled products over the last two minutes is depicted in a bar chart. Further sensor data could enrich this condition monitoring approach. 


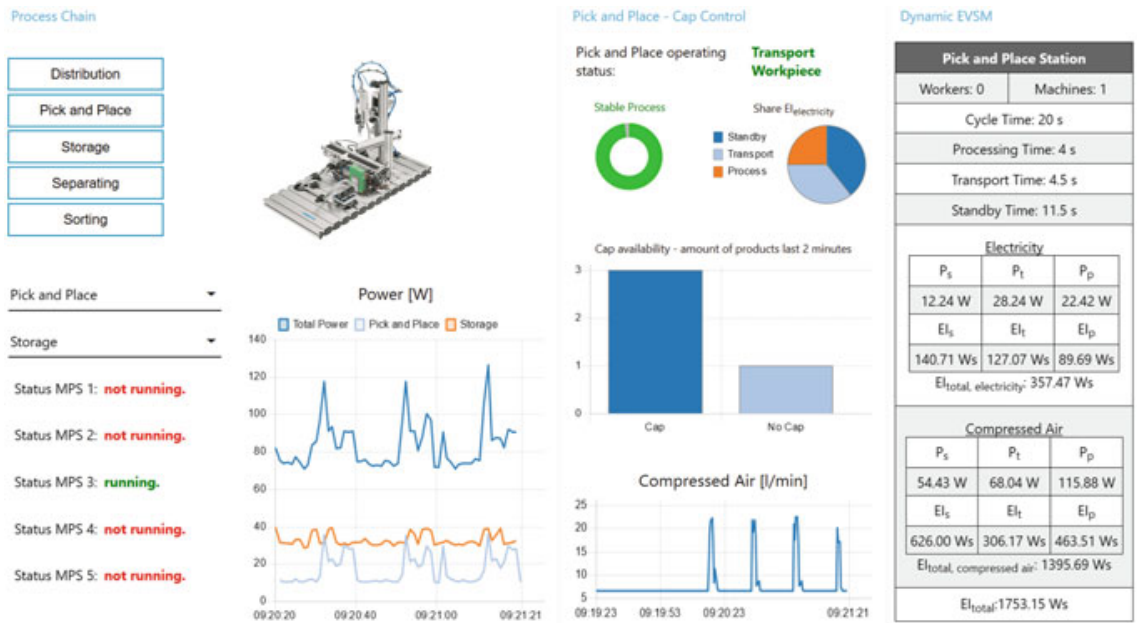

Fig. 11.4 Dashboard of the process and quality monitoring tool including real time data visualisation and data classification showing status report and dynamic Energy Value Stream Mapping

\subsection{Conclusion and Outlook}

In this paper it is shown how a process and quality monitoring tool on the basis of the CPPS framework can be implemented. By using energy data for monitoring of electro-pneumatic automation systems, independence from machine control and scalability can be guaranteed. The exemplary implementation shows that analytics of energy data can be a good way to achieve advanced support for an operator to run an electro-pneumatic production system. By providing an independent data gateway, further processing is not limited. The adaptation of the concept is thus possible also in industrial set-ups.

Acknowledgements The research for this paper is part of the project "JInGEL_Joint Indo German Experience Lab", a joint project between Technische Universität Braunschweig and Birla Institute of Technology and Science Pilani, and is funded by the German Academic Exchange Service (DAAD) under grant number 57219215. The authors are thankful for the funding and the support.

\section{References}

Chakrabarti S et al (2009) Data mining: know it all, 1st edn. Morgan Kaufmann, Elsevier, Amsterdam Larose D, Larose C (2014) Discovering knowledge in data, 2nd edn. Wiley, Hoboken, New Jersey. https://doi.org/10.1002/9781118874059

Lu Y (2017) Industry 4.0: a survey on technologies, applications and open research issues. J Ind Inf Integr. 6:1-10. Elsevier, Amsterdam. https://doi.org/10.1016/j.jii.2017.04.005 
Statista: Umsatz der Festo AG \& Co. KG in den Jahren 2010 bis 2018. https://de.statista.com/ statistik/daten/studie/444500/umfrage/umsatz-von-festo/. Last accessed 21 May 2019

Thiede S, Jurascheck M, Herrmann C (2014) Implementing cyber-physical production systems in learning factories. In: 6th CIRP conference on learning factories, pp 7-12. Elsevier, Amsterdam. https://doi.org/10.1016/j.procir.2016.04.098

Vaidya S, Ambad P, Bhosle S (2018) Industry 4.0-a glimpse. In: Procedia manufacturing, vol 20, pp 233-238. Elsevier, Amsterdam. https://doi.org/10.1016/j.promfg.2018.02.034

Watter H (2017) Hydraulik und Pneumatik, 5th edn. Springer Vieweg, Wiesbaden. https://doi.org/ 10.1007/978-3-658-18555-8

Open Access This chapter is licensed under the terms of the Creative Commons Attribution 4.0 International License (http://creativecommons.org/licenses/by/4.0/), which permits use, sharing, adaptation, distribution and reproduction in any medium or format, as long as you give appropriate credit to the original author(s) and the source, provide a link to the Creative Commons license and indicate if changes were made.

The images or other third party material in this chapter are included in the chapter's Creative Commons license, unless indicated otherwise in a credit line to the material. If material is not included in the chapter's Creative Commons license and your intended use is not permitted by statutory regulation or exceeds the permitted use, you will need to obtain permission directly from the copyright holder.

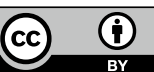

